\title{
A Clinical Observational Study of Effectiveness of a Solid Coupling Medium in Extracorporeal Shock Wave Lithotripsy
}

Hao-Han Chang

Kaohsiung Medical University

Yu-Chih Lin

Kaohsiung Medical University Hospital

Ching-Chia Li

Kaohsiung Medical University Hospital

Wen-Jeng Wu

Kaohsiung Medical University Hospital

Wen-Chin Liou

St. Joseph Hospital Kaohsiung

Yusen Eason Lin

National Kaohsiung Normal University

Wei-Chuan Chen ( $\nabla$ coupe055@ms28.hinet.net)

Kaohsiung Medical University

\section{Research Article}

Keywords: Coupling, Air pockets, Coupling medium, Extracorporeal SW Lithotripter

Posted Date: November 30th, 2021

DOI: https://doi.org/10.21203/rs.3.rs-1039752/v1

License: (9) This work is licensed under a Creative Commons Attribution 4.0 International License.

Read Full License

Version of Record: A version of this preprint was published at BMC Urology on April 12th, 2022. See the published version at https://doi.org/10.1186/s12894-022-01001-y. 


\section{Abstract}

This study aimed to investigate clinical effectiveness of stone disintegration by using isolation coupling pad("icPad") as coupling medium to reduce trapped air pockets during extracorporeal shock wave lithotripsy (ESWL). Patients underwent ESWL between Oct. 2017 to May. 2018 were enrolled in this clinical observational study. An electromagnetic lithotripter (Dornier MedTech Europe GmbH Co., Germany) was used in this study. Patients were divided into icPad group P1, P2 and semi-gel group C by different coupling medium. The energy level and total number of shock wave (SW) for group P1 and C was set at level 2 and 3000 and group P2 at level 3 and 2500. The successful stone disintegration rate (SSDR) was determined to evaluate the treatment outcome. All patients were evaluated by KUB film and ultrasonography after 90 days. Complications during ESWL were recorded. A total of 300 patients satisfied the inclusion criteria. There were no significant differences in characteristics of patients and stone among three groups. The corresponding SSDRs for patients in group P1, P2 and C was 73.0\%, $73.2 \%$ and $55.3 \%$, respectively. The SSDR in group P1 was statistically higher than Group C. Comparing to semi-liquid gel, coupling medium using by icPad could achieve better treatment outcome of stone disintegration in ESWL.

\section{Introduction}

The revolution of extracorporeal shock wave lithotripsy (ESWL) provided an insight into the role of stone treatment in the current and future [1,2]. ESWL had kept its role as a single noninvasive treatment in stone management. Yet, there is still room for improvement in treatment outcome[1, 6]. The evolved works included design of shock wave (SW) generator and focal zone, technical procedure of ramping and lowered pulse rate, target localization and adequate coupling in the past three decades[3-5].

Modality of SW transmission shifting from water bath to water-cushion has made this procedure more convenient and comfortable for patients during ESWL procedure. However, the effectiveness were not comparable to the original one[7, 8]. The reason was inadequate coupling because air pockets trapped during smearing semi-liquid gel could impair the acoustic energy transmission of SW and then significantly decreased effectiveness of stone disintegration [9]. For instance, air pockets covering 1.5 $19 \%$ of coupling area would reduce amplitude reduction of $20 \%$ in SW and even $2 \%$ air coverage could decrease stone disintegration rate by $20-40 \%[10]$. Adequate coupling became the major concern to achieve successful outcome in ESWL[11]. Resolution of coupling became a critical step to prevent transmission of SW from acoustic energy loss $[12,13]$.

Regarding coupling with less entrapped air pockets during the procedure, several methods were introduced. First, a large volume of gel dispensing directly onto the head of lithotripter could diminish the amount of entrapped air pockets [10]. This technique using applying a bolus of gel to the treatment head might remove the air bubbles in an in vitro study [14]. Second, the Optical Coupling Control (OCC) system, which equipped with an inline camera for air pockets observation, could help operator to repeat the coupling procedure and achieve less air-pockets coupling $[15,16][17]$. Third, coupling a solid gel disc 
would cause less air bubbles as an alternative option $[18,19]$. Further, a proprietary isolation-coupling pad ("icPad") had demonstrated its superior efficacy of stone disintegration by markedly reducing trapped air pockets during coupling in a phantom study [20]. Given the advantages of icPad's efficacy in stone disintegration, the aim of this study was to further investigate the clinical effectiveness for stone disintegration in patients undergoing ESWL.

\section{Materials \& Methods}

During Oct. 2017 to May. 2018, patients with ureteral or renal stones were eligible for this study by KUB, ultrasonography or non-enhanced computed tomography. An electromagnetic lithotripter (Dornier MedTech Europe GmbH Co., Germany) was used. The coupling medium were icPads (Diameter=150 mm, Thickness $=8 \mathrm{~mm}$ ) consisting of chemical-gel, mainly polyacrylamide (Fig. 1) and standard semi-liquid gel (Sonogel $\circledast$ ) widely used in clinical practice. The procedure of applying icPad was demonstrated in figure 2 (Fig. 2).

A total of 300 patients were enrolled in this study. 100 patients were treated in the Group P1, 97 patients in the Group P2, and 103 in Group C. The patients were divided into three groups (Group P1, P2 and C) according to different coupling medium (icPad or semi-liquid gel) and lithotripsy settings (energy lever and total number of SW)(Table 1). All treatments were performed by attending urologists and assisted by an experienced nurse. Before the treatment, patient's medical history, physical examination, urine analysis and radiologic investigation were performed. characteristics patients and treated stones were recorded. Stone free (SF) was defined as complete absence of stone fragments and clinical insignificant residual disintegration (CIRD) was defined as stone burden less than $4 \mathrm{~mm}$ on KUB examination after ESWL. Successful stone disintegration rate (SSDR) of each group was calculated as the patients of SF and $\mathrm{CIRD} /$ total patients of each group and was used to evaluate the treatment effectiveness. All patients were evaluated by KUB film and ultrasonography 90 days after ESWL. Complications were recorded during or after ESWL.

Table 1

Treatment parameters of ESWL

\begin{tabular}{|llll|}
\hline & $\begin{array}{l}\text { Group C } \\
\text { N=103 }\end{array}$ & $\begin{array}{l}\text { Group P1 } \\
\text { N=100 }\end{array}$ & $\begin{array}{l}\text { Group P2 } \\
\text { N=97 }\end{array}$ \\
\hline Coupling medium & Semi-liquid gel & icPad & icPad \\
\hline Total SW numbers/session & 3000 & 3000 & 2500 \\
\hline Rate of pulse of hock (number/min.) & 90 & 90 & 90 \\
\hline Energy level & 3 & 3 & 3 \\
\hline Fluoroscopy time(sec.) & $347.1 \pm 159.2$ & $370.9 \pm 158.1$ & $311.3 \pm 114.4$ \\
\hline Treatment time(min.) & $39.36 \pm 3.48$ & $39.55 \pm 3.79$ & $41.53 \pm 3.13$ \\
\hline
\end{tabular}


Chi-square test, one-way ANOVA were used for categorical and numerical variables. Statistical significance was set at $p<0.05$. IBM SPSS 26.0 (IBM Corp., Armonk, NY) was used for all statistical analyses.

\section{Results}

There were no significant differences in characteristics of patients and treated stones among three groups (Table 2). The chi-square test revealed that treatment outcomes (SSDR) were significantly different between group P1 and group C (73.0\% vs. 55.3\%, $p=0.009)$, but not significantly different between group P1 and P2( $p=0.975)$ (Table 3$)$. No major organ complications were noted in each group.

Table 2

Patients' and Stones' characteristics

\begin{tabular}{|c|c|c|c|c|c|}
\hline Parameters & Group C & Group P1 & Group P2 & F value & $P$ value \\
\hline Number of patients & 103 & 100 & 97 & & \\
\hline Age (years) & $52.5 \pm 12.4$ & $51.4 \pm 11.1$ & $50.4 \pm 10.9$ & 0.845 & 0.431 \\
\hline Stone size $(\mathrm{mm})$ & $8.14 \pm 3.46$ & $7.94 \pm 2.84$ & $8.14 \pm 3.12$ & 0.134 & 0.875 \\
\hline \multicolumn{6}{|l|}{ Stone side (No./\%) } \\
\hline Left & $59(57.3 \%)$ & $60(60.0 \%)$ & $56(57.7 \%)$ & & \\
\hline Right & $44(42.7 \%)$ & $40(40.0 \%)$ & $41(42.3 \%)$ & & \\
\hline \multicolumn{6}{|c|}{ Stone location(No./\%) } \\
\hline Kidney & $47(45.6 \%)$ & $50(50.0 \%)$ & $47(48.5 \%)$ & & \\
\hline Upper ureter & $25(24.3 \%)$ & $28(28.0 \%)$ & $24(24.7 \%)$ & & \\
\hline Middle ureter & $4(3.9 \%)$ & $0(0.0 \%)$ & $3(3.1 \%)$ & & \\
\hline Lower ureter & $27(26.2 \%)$ & $22(22.0 \%)$ & $23(23.7 \%)$ & & \\
\hline
\end{tabular}


Table 3

Successful stone disintegration rate (SSDR) of each treatment the groups

\begin{tabular}{|c|c|c|c|c|c|c|c|}
\hline & \multicolumn{2}{|c|}{$\leq 4 \mathrm{~mm}$} & \multicolumn{2}{|c|}{$>4 \mathrm{~mm}$} & \multirow[t]{2}{*}{ total } & \multirow[t]{2}{*}{$x^{2}$} & \multirow[t]{2}{*}{$p$-value } \\
\hline & $\mathrm{n}$ & $\%$ & $\mathrm{n}$ & $\%$ & & & \\
\hline Group C & 57 & 55.3 & 46 & 44.7 & 103 & 6.872 & .009 \\
\hline \multirow[t]{3}{*}{ Group P1 } & 73 & 73.0 & 27 & 27.0 & 100 & & \\
\hline & \multicolumn{2}{|c|}{$\leq 4 \mathrm{~mm}$} & \multicolumn{2}{|c|}{$>4 \mathrm{~mm}$} & total & $x^{2}$ & $P$ \\
\hline & $\mathrm{n}$ & $\%$ & $\mathrm{n}$ & $\%$ & & & \\
\hline Group P1 & 73 & 73.0 & 27 & 27.0 & 100 & .001 & .975 \\
\hline Group P2 & 71 & 73.2 & 26 & 26.8 & 97 & & \\
\hline
\end{tabular}

\section{Discussion}

In 1983, the first lithotripter became available to treat urinary stones extracorporeally. It was discovered that there was very low energy dissipation when SW energy traveled through water[21]. The coupling system in this lithotripter provided excellent SW energy transmission, yet, the patient was required to be submerged in a water tank[22]. The procedure was inconvenient and made patients uncomfortable. Later, coupling in dry head lithotripsy the was invented and could make the patient positioned on a table without wetting the whole body. However, it did not provide the similar effectiveness due to decreased energy of transmitted SW[23, 7]. The effectiveness of different coupling design revealed that SW transmission through water could provide better outcome of stone disintegration[7, 23, 8]. The reason is the presence of "trapped air pockets" in coupling could reduce the acoustic transmission of energy by reflecting SW. Therefore, removing the air pockets in coupling gel were pivotal for effectiveness of stone disintegration[24, 16, 25].

Both techniques of applying a bolus of gel and assistance of OCC were introduced to decrease the presence of the entrapped air pockets during coupling.[26, 10]. Better SFR as well as less total numbers were demonstrated in the patients undoing ESWL by the assistance of OCC[19]. However, smearing larger gel was still an operator-dependent technique and the higher cost using OCC might hinder its wide use by healthcare providers. In our previous phantom study, icPads had demonstrated better coupling and higher efficacy of stone disintegration than semi-liquid gel (92.3\% vs. 45.5\%) [20]. The area of trapped air pockets observed in coupling using icPad was only $0.38 \%$, which was significantly lower than that of semi-liquid gel (2.55\%). Even after sliding patient on the treatment table during ESWL procedure, air pockets only increased as little as $0.54 \%$. Given the superior efficacy of icPad in stone disintegration in our phantom study[20], this study aimed to further investigate the clinical effectiveness for stone disintegration by lowering the total number of SW to 2500 in group P2. The SSDR of group P1 or P2 
(73.0\% vs. $73.2 \%$ ) was higher than group C (55.3\%). After running Chi-square test for comparison SSDR (stone burden $\leq 4 \mathrm{~mm}$ ) among 3 groups, the results demonstrated that treatment outcome using icPad was better than semi-liquid and lowered total number of SW could achieve the similar outcome in icPad groups. It also indicated that the work life of lithotripter generator might be prolonged by applying less number of SW.

This is the first clinical observational study to investigate the effectiveness of the newly designed icPad. However, the study was conducted at single center and further studies are needed to validate our results.

\section{Conclusions}

To our knowledge, this is the first clinical observational study to investigate the effectiveness of coupling medium using a solid gel pad during ESWL procedure. The advantages of icPad demonstrated that similar patient outcome of stone disintegration could be achieved at lower number of SW and energy level. Our encouraging results suggested that the icPad as a coupling medium might be a cost-effective solution for future lithotripters.

\section{Declarations}

\section{Compliance with Ethical Standards}

This study was approved by the Institutional Review Board of Kaohsiung Medical University Hospital (KMUHIRB-E(II)-20200410) conducting in accordance with the Declaration of Helsinki. As this work is a retrospective, case-control study, this study is waiver of subject informed consent approving by the Institutional Review Board

\section{Conflict of Interest}

All the authors declare that they have no competing interests. The company Clean Wave Medical did not provide any funding nor financial incentives to authors expect donating free icPad for this study. All procedures performed in studies involving human participants were in accordance with the ethical standards of the institutional and/or national research committee and with the 1964 Helsinki declaration, and its later amendments or comparable ethical standards.

\section{Ethics approval and consent to participate}

This study was approved by the Institutional Review Board of Kaohsiung Medical University Hospital (KMUHIRB-E(II)-20200410) conducting in accordance with the Declaration of Helsinki. As this work is a retrospective, case-control study, this study is waiver of subject informed consent approving by the Institutional Review Board of Kaohsiung Medical University Hospital

IRB Number: KMUHIRB-E(II)-20200410 


\section{Consent for publication}

Not applicable

\section{Availability of data and materials}

All data generated or analysed during this study are included in this published article

\section{Competing interests}

All the authors declare that they have no conflict of interest.

\section{Funding}

The authors declare that they have no funding

\section{Authors' contributions}

Chang Hao-Han wrote the main manuscript text and prepared all the figures and tables

Lin Yusen Eason checked the grammar and spelling of the manuscript

Lin Yu-Chih did the statistics analysis

Liou Wen-Chin, Li Ching-Chia and Wu Wen-Jeng helped with data and patient collection

Chen Wei-Chuan was the corresponding author, came up with the main idea

All authors reviewed the manuscript

\section{Acknowledgments}

Not applicable

\section{References}

1. Basulto-Martinez M, Klein I, Gutierrez-Aceves J (2019) The role of extracorporeal shock wave lithotripsy in the future of stone management. Curr Opin Urol 29 (2):96-102. doi:10.1097/MOU.0000000000000584

2. Tsu-Ming Chien Y-ML, Yii-Her Chou, Wen-Jeng Wu, Chun-Nung Huang (2017) Shock wave lithotripsy for renal stones is not associated with development of hypertension in Taiwan's Chinese population. Urological Science 28 (4):219-222. doi:10.1016/j.urols.2016.05.004

3. Rassweiler J, Rassweiler M-C, Frede T, Alken PJljouljotUSol (2014) Extracorporeal shock wave lithotripsy: An opinion on its future. Indian J Urol 30 (1):73 
4. Elmansy H, Lingeman JJIJoS (2016) Recent advances in lithotripsy technology and treatment strategies: A systematic review update. Int J Surg 36:676-680

5. Po-Cheng Chen Y-TL, Jui-Hsiang Hsieh, Chung-Cheng Wang (2017) A practical formula to predict the stone-free rate of patients undergoing extracorporeal shock wave lithotripsy. Urological Science 28 (4):215-218. doi:10.1016/j.urols.2016.05.004

6. Basulto-Martínez M, Klein I, Gutiérrez-Aceves JJCoiu (2019) The role of extracorporeal shock wave lithotripsy in the future of stone management. Curr Opin Urol. 29 (2):96-102

7. Gerber R, Studer UE, Danuser H (2005) Is newer always better? A comparative study of 3 lithotriptor generations. J Urol 173 (6):2013-2016. doi:10.1097/01.ju.0000158042.41319.c4

8. Ng CF, McLornan L, Thompson TJ, Tolley DA (2004) Comparison of 2 generations of piezoelectric lithotriptors using matched pair analysis. J Urol 172 (5 Pt 1):1887-1891.

doi:10.1097/01.ju.0000142730.60583.7a

9. Lawler AC, Ghiraldi EM, Tong C, Friedlander JIJCur (2017) Extracorporeal shock wave therapy: current perspectives and future directions. Curr Urol Rep. 18 (4):25

10. Neucks JS, Pishchalnikov YA, Zancanaro AJ, VonDerHaar JN, Williams JC, McAteer JAJUr (2008) Improved acoustic coupling for shock wave lithotripsy. Urol Res. 36 (1):61-66

11. Zhong P Innovations in lithotripsy technology. In: AIP Conference Proceedings, 2007. vol 1. American Institute of Physics, pp 317-325

12. Jain A, Shah TKJEu (2007) Effect of air bubbles in the coupling medium on efficacy of extracorporeal shock wave lithotripsy. Eur Urol. 51 (6):1680-1687

13. Jin Kyu Kim1 MEC, Stephen Shei-Dei Yang3, Jessica M Ming4, Joana Dos Santos2, Walid A Farhat5 (2020) Recent literature on the minimally invasive management of pediatric urolithiasis: A narrative review. Urological Science 31 (6):244-252. doi:10.4103/UROS.UROS_91_20

14. Duryea AP, Roberts WW, Cain CA, Tamaddoni HA, Hall TLJJoe (2014) Acoustic bubble removal to enhance SWL efficacy at high shock rate: An in vitro study. ? 28 (1):90-95

15. Elmansy HE, Lingeman JE (2016) Recent advances in lithotripsy technology and treatment strategies: A systematic review update. Int J Surg 36 (Pt D):676-680. doi:10.1016/j.ijsu.2016.11.097

16. McClain PD, Lange JN, Assimos DG (2013) Optimizing shock wave lithotripsy: a comprehensive review. Rev Urol 15 (2):49-60

17. Tailly GG, Tailly-Cusse MMJJoe (2014) Optical coupling control: an important step toward better shockwave lithotripsy. J Endourol. 28 (11):1368-1373

18. Bergsdorf T, Chaussy C, Thueroff S. 1815 Coupling gel viscosity-a relevant factor for efficient shock wave coupling in SWL. J Urol. 2010;183(4S):e704-e704.

19. Lv JL (2016) A new optical coupling control technique and application in SWL. Urolithiasis 44 (6):539-544. doi:10.1007/s00240-016-0874-9

20. Wang CS, Li CC, Wu WJ, Liou WC, Lin YE, Chen WC (2021) Newly designed solid coupling medium for reducing trapped air pockets during extracorporeal shock wave lithotripsy_ a phantom study. BMC 
Urol 21 (1):79. doi:10.1186/s12894-021-00847-y

21. Delius M (2003) Extracorporeal Shock Waves: Bioeffects and Mechanisms of Action.211-226

22. Pishchalnikov YA, Neucks JS, VonDerHaar RJ, Pishchalnikova IV, Williams JC, McAteer JAJTJou (2006) Air pockets trapped during routine coupling in dry head lithotripsy can significantly decrease the delivery of shock wave energy. J Urol. 2006 Dec;176(6 Pt 1):2706-10.

23. Graber SF, Danuser H, Hochreiter WW, Studer UE (2003) A prospective randomized trial comparing 2 lithotriptors for stone disintegration and induced renal trauma. J Urol 169 (1):54-57

24. Jain A, Shah TK (2007) Effect of air bubbles in the coupling medium on efficacy of extracorporeal shock wave lithotripsy. Eur Urol 51 (6):1680-1686; discussion 1686-1687. doi:10.1016/j.eururo.2006.10.049

25. Rassweiler JJ, Knoll T, Kohrmann KU, McAteer JA, Lingeman JE, Cleveland RO, Bailey MR, Chaussy C (2011) Shock wave technology and application: an update. Eur Urol 59 (5):784-796. doi:10.1016/j.eururo.2011.02.033

26. Bohris C, Roosen A, Dickmann M, Hocaoglu Y, Sandner S, Bader M, Stief CG, Walther S (2012) Monitoring the coupling of the lithotripter therapy head with skin during routine shock wave lithotripsy with a surveillance camera. J Urol 187 (1):157-163. doi:10.1016/j.juro.2011.09.039

\section{Figures}




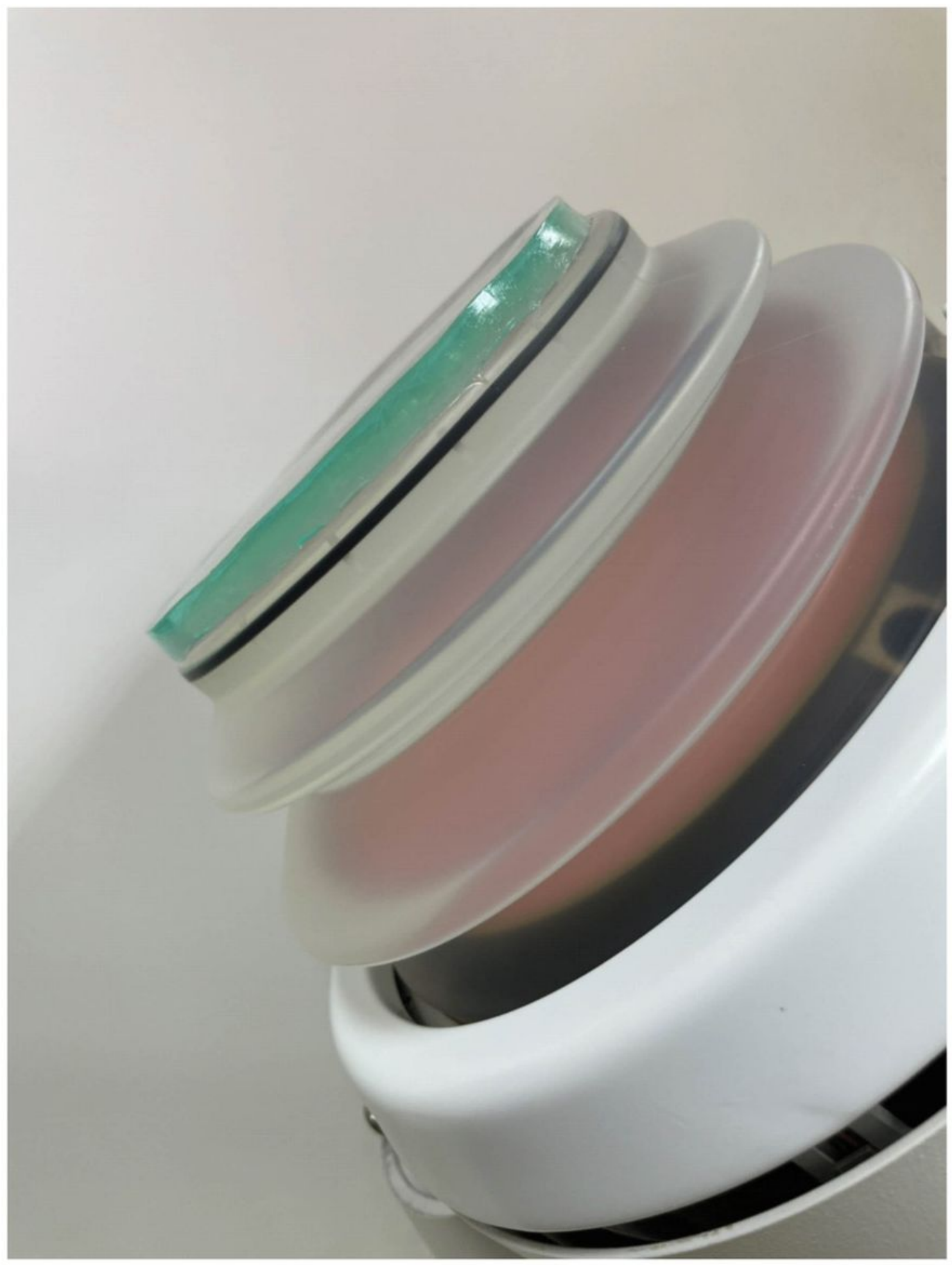

Figure 1

A proprietary IcPad (blue color) fit tightly on the treatment head. 

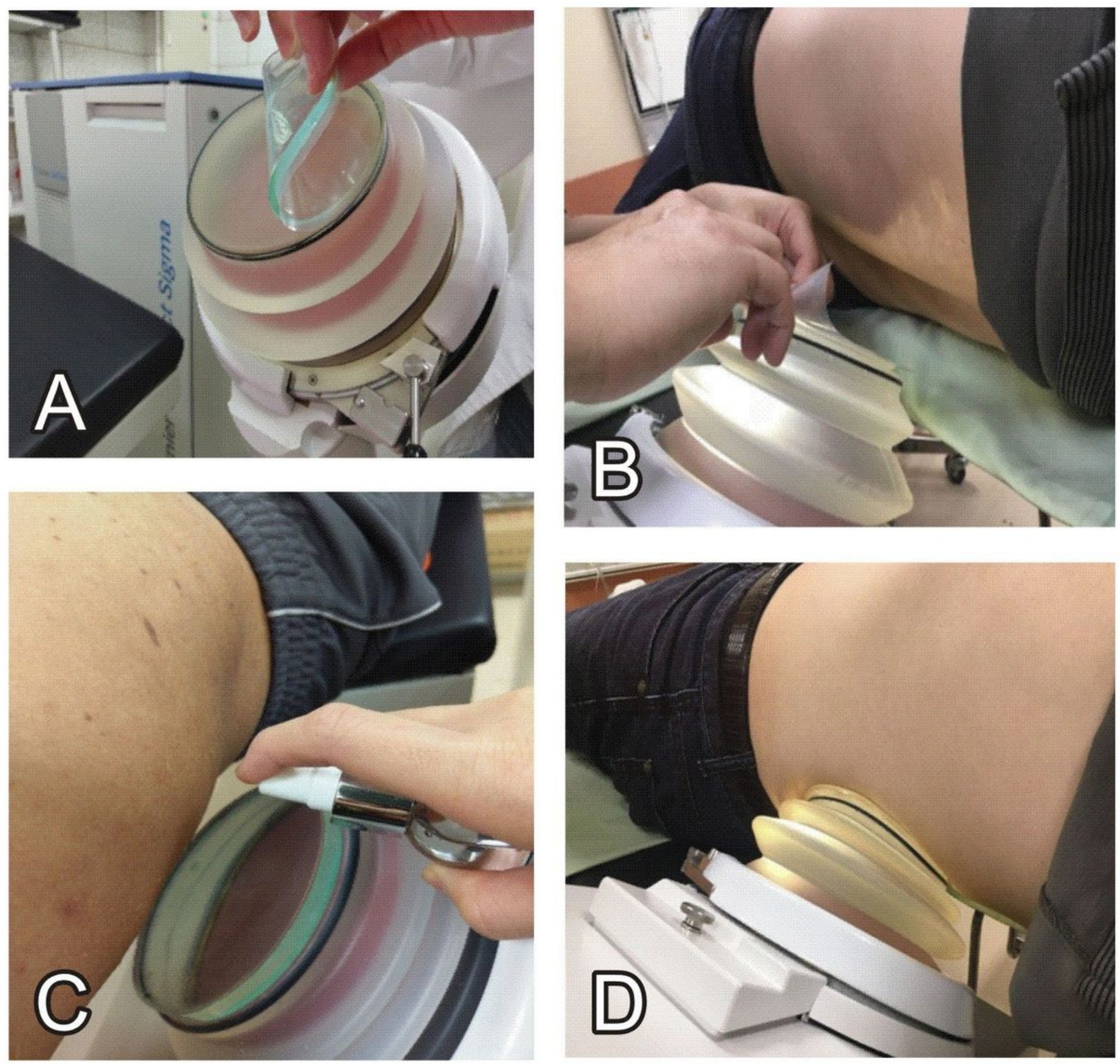

Figure 2

The procedure of applying icPad. (A) Paste the icPad gently to the head of lithotripter (B) Remove the cover on the body side of icPad following probe side pasted. (C) Spray lubricant to surface of body side before moving bellow to the body. (D) Complete coupling before starting ESWL. The whole procedure can be completed in about two minutes. 